\title{
Neuro-ocular vestibular dysfunction: an overlooked illness
}

\begin{abstract}
Neuro-ocular vestibular dysfunction is an illness involving the neurologic, ocular and vestibular systems. It includes the signs of motion sickness such as nausea, headache, and/or dizziness, and photophobia or visual sensitivity to motion and headache. It is usually overlooked in the standard eye examination. To know the main features of neuroocular vestibular dysfunction may be beneficial for ophthalmologists, neurologists, and otolaryngologists in the diagnosis of this disorder to be able to overlook.
\end{abstract}

Keywords: see-sick syndrome, motion sickness, visually related, neuro-ocular vestibular dysfunction, nausea, head-ache, dizziness, photophobia
Volume 7 Issue 7 - 2017

Burak Turgut, Fatoș Altun Turgut

Department of Ophthalmology, Yuksek Ihtisas University, Turkey

Correspondence: Burak Turgut, Professor of Ophthalmology, Yukseklhtisas University, Faculty of Medicine, Department of Ophthalmology, 06520, Ankara, Turkey, Tel +90 312 280360I, Fax +90 312 2803605, Email burakturgut@yiu.edu.tr

Received: December 25, 2017 | Published: December 28, 2017
Abbreviations: NOVD, neuro-ocular vestibular dysfunction; SSS, see-sick syndrome

\section{Editorial}

Neuro-ocular vestibular dysfunction (NOVD) or see-sick syndrome (SSS) is a condition arising from the hypersensitivity to visual motion and commonly associated with motion sickness, headaches, and sensitivity to light (photophobia). NOVD occurs in case of the disconnection between vision (ocular) and balance (vestibular) systems. NOVD usually occurs during the traveling/ riding or reading on a plane, bus, car, ship or any transporting vehicle. However, it may also experience while watching moving objects as seen sporting events, television or three dimensioned movies or other social activities and the walking in crowded places or while shopping in the supermarket. Moreover, NOVD is called sometimes as "supermarket disorder/syndrome". NOVD occurs in almost two-thirds of the population. In ophthalmological literature, the publications on SSS or NOVD are limited. Already, it is a disorder which has not been exactly understood. ${ }^{1-5}$ It has been mentioned by Dr. Gillilan that NOVD is seen in almost $6 \%$ of the females and $1 \%$ of the males in different severity levels. ${ }^{1}$ Additionally, it has been considered that it is usually genetically. NOVD may seem in all age groups. It tends to be usually in mild level in children and in more severe level in adults. This is probably caused by the avoiding of the symptomproducing activities in adults. Additionally, adult-onset disease can be caused by a result of head trauma. ${ }^{1-5}$ While the brain perceives the position and movements of the body, the inner ear (labyrinth) analyses the data from rotation movements and the movements towards back and forth, sideways and up and down directions. At the same moment, the eyes analyze the position of the body in the environment such as upside down or right side and the data obtained by the observing the direction of formed movement while the sensory receptors in feet, skin, muscles, and joints analyze which part of the body is below and where it touches. ${ }^{1-5}$ It has been believed that the NOVD results from a non-adaptable sensory-perceptual discordance or disconnection with a motion stimulus of visual, vestibular and deep sensory (somatosensory) systems. This perceptual contrast reveals NOVD in people with sensitivity. For example, while sitting in a vehicle, if a vehicle moves, the receptors in the inner ear and deep sensory receptors will perceive the momentum and vibrations of the movement, and they transmit a motion to the brain. However, the visual system will give to the brain the information that there is no any movement. Thus, a conflict regarding with the condition in the brain occurs. ${ }^{1-5}$ It has not been cleared that the exact pathogenesis of the NOVD. However, it is thought that the disorder does not occur without motion detection organs (labyrinth) located in the inner ear while motion sickness can develop blind individuals. Thus, it seems that the visual system might play a lesser role than the inner ear in this disorder. ${ }^{1-5}$

The severe NOVD patients have usually in different degrees at least those symptoms including motion sickness with repeated eye movement or when observing rapid motion; unusual sensitivity to light or photophobia; frequent or constant headaches. In almost a half of the patients with NOVD, a reduced awareness of objects in the periphery (tunnel vision) occurs as a possible protective mechanism. This may cause the klutziness in these subjects and the loss of their place during reading. NOVD usually occurs especially in the daytimes because less motion is observed at the sides of the road at night. ${ }^{1-5}$ The severity and frequency of the symptoms of NOVD may show a wide variation from patient to patient. Some conditions including hormonal alterations such as pregnancy, oral contraceptives and premenstrual syndrome, fatigue, migraine, hypoglycemia and inner ear disorders may worse NOVD. The vast majority of the patients with NOVD have the complaints of the motion sickness such as nausea, headache, dizziness, fatigue, malaise, drowsiness, depression, bodily warmth, pallor, and/or cold sweats during repeated eye movements or observation or pursuit of the rapid motions, and sometimes photophobia. The symptoms usually are ceased if the movement is interrupted. However, even if the stimuli finishes, the complaints in some cases may continue for the days. ${ }^{1-5}$

NOVD is most commonly diagnosed on the basis of patient history and especially by patient's himself/herself which learned the symptoms of the NOVD. NOVD patients have fairly high intelligence level. The disease cannot be diagnosed during a standard ophthalmological examination without the patient complaints regarding abovementioned activities. The presence of only a few symptoms cannot provide to diagnose the NOVD. However, the subject having the 
major symptoms such as an equilibrium problem and difficulty looking at motion is probably a patient having NOVD. Subjects experienced NOVD rarely admits to an ophthalmologist. They often visit a lot of doctors including the family physician, neurologist, vestibular specialist and otolaryngologist to explore their problems. Moreover, they may sometimes be called as crazy or complainer by friends and family members. Additionally, all of these physicians declare the patient that there is nothing wrong or problem. Already, it has been demonstrated that vertigo is an underestimated symptom of the visual disorders. ${ }^{1-6}$ The patients with mild NOVD cannot read and look down in a car more than a few seconds without nausea, headache, and/or dizziness. The cases having moderate NOVD are needed to be the driver to avoid nausea while traveling in a car, even on a straight road and they have the inability to look backward in a car without nausea. They also experience nausea when driving on tree-lined roads and shadowing the sun casts across the road. In severe NOVD cases, severe headache, fatigue, and/or nausea occur when riding or even driving a car for more than a few minutes..$^{1-5}$ In mild NOVD cases, unusual sensitivity to light to a moderate degree is present. They have to wear sunglasses outdoors, even on cloudy days. They feel uncomfortable in brightly lit buildings or places with shiny floors such as grocery stores, classrooms, offices, or malls. In moderate cases, ocular pain or a headache occurs when they exposed to a "flash of light" or being outside without sunglasses, even on cloudy days, or being in a brightly lit place. They can sometimes experience longterm after images lasting several minutes after looking at most sources of light. Computer screen brightness causes also discomfort in these patients. In some subjects, the sensation of being "blinded" when looking at oncoming headlights can occur. In cases with severe NOVD, there is severe sensitivity to light both indoors and out. These cases are individuals turning off lights and pulling-down shades and which consider that light is their worst enemy. ${ }^{1-5}$ The subjects with mild NOVD cannot sit close to a movie screen or watch the movement of a train or a carnival ride without nausea, headache, and/or dizziness. Moderate cases have the inability to look at stripes or watch rapid movement on television or a moving belt without nausea, headache, or dizziness. Severe cases having NOVD cannot watch more than minimal motion without dizziness or nausea, such as watching one's own hand while eating. ${ }^{1-5}$ In mild NOVD cases, headache as the type of compression feeling may sometimes occur. Moderate NOVD cases experience severe headaches which may last a few days when they did any activities involving the observations of the movement or eye movement for more than a few minutes. In severe NOVD cases, constant or very frequent nausea, headache, or dizziness ranging from mild to severe is seen. ${ }^{1-5}$

Some recommendations for the prevention and the relief of NOVD are available. Whatever the vehicle, it is recommended for the avoidance of the heavy and greasy food during the journey. It should always sit in a place where the movement of the body can be perceived in the same way by inner ear and eyes. ${ }^{6-9}$ When traveling in a car, the choosing to travel during the daytime, the sitting on the front passenger seat of the car and looking at the landscape, opening the windows to get fresh air, avoiding reading books/newspapers can be beneficial. When traveling in a bus, addition to the recommendations for the car traveling, the sitting on the seats on the wheels of the vehicle should be avoided because these seats will shake the subject more. Additionally, the seats opposite to the travel direction should not be chosen. In a ship traveling, while the ship is moving, being situated in the middle parts of the deck and looking at the horizon line will be beneficial. In-plane travel, sitting on just near the window, choosing the chairs fitting on the wing which has the least amount of movement may be beneficial. ${ }^{6-9}$ In the brain, the data from the surround organs functions through neurotransmitters such as histamine, acetylcholine, and norepinephrine. Most drugs used in NOVD acts as the influencing the level of these substances and the suppressing the brain regions where the signals are processed or by controlling nausea. These medications include antihistaminic drugs, serotonergic drugs, anticholinergic drugs, benzodiazepines, antidopaminergic drugs and sympathomimetics. ${ }^{7-11}$ However, these medications can provide a partial impairment in the symptoms. Dynamic adaptive vision or optometric therapy may be an effective treatment for patients diagnosed with the NOVD. This therapy is based on the principle of neuroplasticity and the exercises re-connecting between the eye and brain the learning of new visual and motional skills. ${ }^{12,13}$ However, the number of optometrists or ophthalmologist training this disease and management is very low.

\section{Conclusion}

NOVD or SSS is a disorder which is often overlooked in the standard eye examination. Currently, there is no detailed publication on NOVD in ophthalmological literature. The understanding of NOVD may be beneficial for ophthalmologists, neurologists, and otolaryngologists in the diagnosis of this disorder to be able to overlook.

\section{Acknowledgments}

None.

\section{Funding}

The authors received no financial support for the research, authorship, and/or publication of this article.

\section{Conflicts of interest}

The authors declare that there is no conflict of interest regarding the publication of this paper.

\section{Authorship contributions}

Concept and Design, Data Collection, Analysis, Interpretation: Burak Turgut and Fatoş Altun Turgut; Literature Search: Fatoş Altun Turgut; Writing: Burak Turgut.

\section{References}

1. http://www.seesicksyndrome.com/wp-content/uploads/2014/10/ SeeSickSyndromeManual.pdf

2. Jay WM, Jay MS. Vision and motion sickness. J Pediatr Ophthalmol Strabismus. 1981;18(6):34-37.

3. Golding JF. Motion sickness. Handb Clin Neurol. 2016;137:371-390.

4. Flanagan MB, May JG, Dobie TG. The role of vection, eye movements and postural instability in the etiology of motion sickness. $J$ Vestib Res. 2004;14(4):335-346.

5. Bertolini G, Straumann D. Moving in a Moving World: A Review on Vestibular Motion Sickness. Front Neurol. 2016;7:14.

6. Zhang LL, Wang JQ, Qi RR, et al. Motion Sickness: Current Knowledge and Recent Advance. CNS Neurosci Ther. 2016;22(1):15-24.

7. Murdin L, Golding J, Bronstein A. Managing motion sickness. BMJ. 2011;343:7430 
8. Shupak A, Gordon CR. Motion sickness: advances in pathogenesis, prediction, prevention, and treatment. AviatSpace Environ Med. 2006;77(12):1213-1223.

9. Brainard A, Gresham C. Prevention and treatment of motion sickness. Am Fam Physician. 2014;90(1):41-46.

10. Marcus DA, Furman JM. Prevention of motion sickness with rizatriptan: a double-blind, placebo-controlled pilot study. Med Sci Monit. 2006;12(1):PI1-PI7.
11. Strupp M, Brandt T. Current Treatment of Vestibular, Ocular Motor Disorders and Nystagmus. Ther Adv Neurol Disord. 2009;2(4):223-239.

12. Golding JF, Gresty MA. Pathophysiology and treatment of motion sickness. Curr Opin Neurol. 2015;28(1):83-88.

13. Gillilan RW, Todd D. Vision therapy as a treatment for motion sickness. $J$ Am Optom Assoc. 1986;57(6):456-458. 